\title{
Electromechanical Analysis of a Ring-type Piezoelectric Transformer
}

\author{
Shine-Tzong Ho \\ Kaohsiung University of Applied Sciences
}

Taiwan

\section{Introduction}

The idea of a piezoelectric transformer (PT) was first implemented by Rosen (Rosen, 1956), as shown in Fig.1. It used the coupling effect between electrical and mechanical energy of piezoelectric materials. A sinusoidal signal is used to excite mechanical vibrations by the inverse piezoelectric effect via the driver section. An output voltage can be induced in the generator part due to the direct piezoelectric effect. The PT offers many advantages over the conventional electromagnetic transformer such as high power-to-volume ratio, electromagnetic field immunity, and nonflammable.

Due to the demand on miniaturization of power supplying systems of electrical equipment, the study of PT has become a very active research area in engineering. In literatures (Sasaki, 1993; Bishop, 1998), many piezoelectric transformers have been proposed and a few of them found practical applications. Apart from switching power supply system, a Roson-type PT has been adopted in cold cathode fluorescent lamp inverters for liquid-crystal display. The PT with multilayer structure to provide high-output power may be used in various kinds of power supply units. Recently, PT of ring (Hu, 2001) or disk (Laoratanakul, 2002) shapes have been proposed and investigated. Their main advantages are simple structure and small size. In comparing with the structure of a ring and a disk, the PZT ring offers higher electromechanical coupling implies that a ring structure is more efficient in converting mechanical energy to electrical energy, and vice versa, which is essential for a high performance PT.

Different from all the conventional PT, the ring-type PT requires only a single poling process and a proper electrode pattern, and it was fabricated by a PZT ring by dividing one of the electrodes into two concentric circular regions. Because of the mode coupling effect and the complexity of vibration modes at high frequency, the conventional lumped-equivalent circuit method may not accurately predict the dynamic behaviors of the PT.

In this chapter, an electromechanical model for a ring-type PT is obtained based on Hamilton's principle. In order to establish the model, vibration characteristics of the piezoelectric ring with free boundary conditions are analyzed in advance, and the natural frequencies and mode shapes are obtained. In addition, an equivalent circuit model of the PT is obtained based on the equations of the motion for the coupling electromechanical system. Furthermore, the voltage step-up ratio, input impedance, output impedance, input 
power, output power, and efficiency for the PT will be conducted. Then, the optimal load resistance and the maximum efficiency for the PT will be calculated.

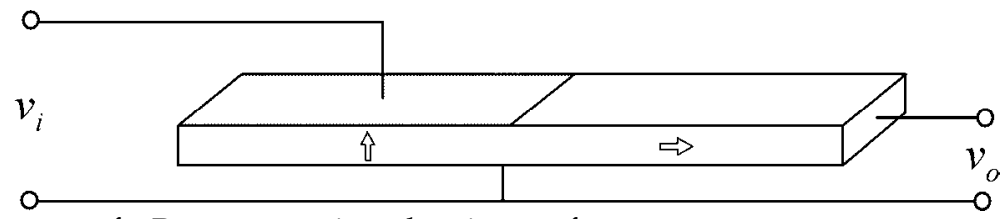

Fig. 1. Structure of a Rosen-type piezoelectric transformer.
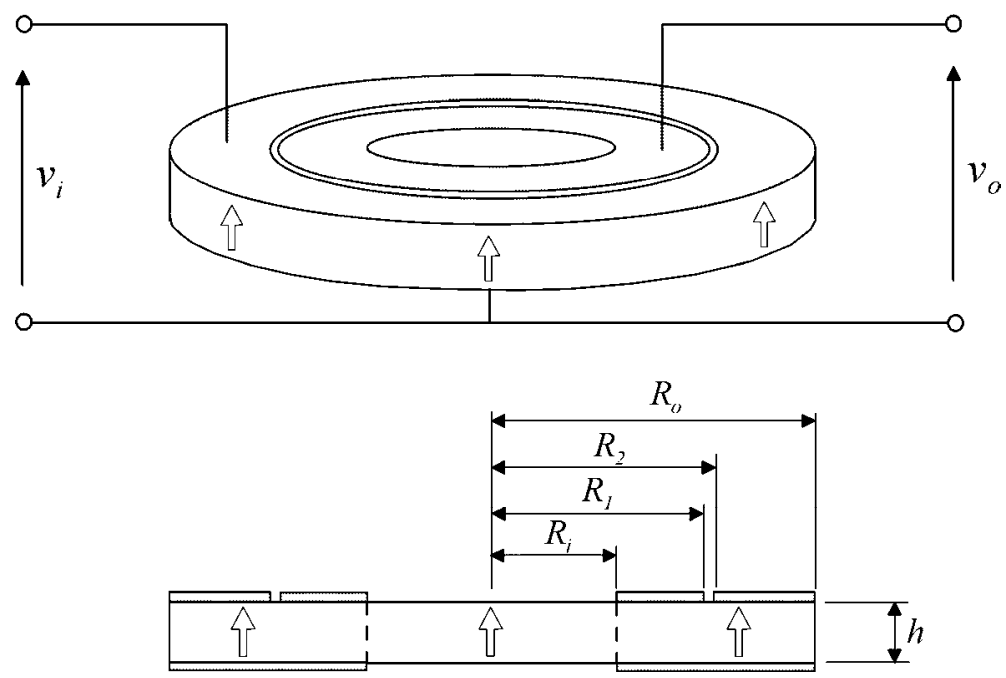

Fig. 2. Structure of a ring-type piezoelectric transformer.

\section{Theoretical Analysis}

\subsection{Vibration Analysis of the Piezoelectric Ring}

Fig.2 shows the geometric configuration of a ring-type PT with external radius $R_{o}$, internal radius $R_{i}$, and thickness $h$. The ring is assumed to be thin, $h<<R_{i}$. The cylindrical coordinate system is adopted where the $r-\theta$ plane is coincident with the mid-plane of the undeformed ring, and the origin is in the center of the ring. The piezoelectric ring is polarized in the thickness direction, and two opposite surfaces are covered by electrodes. The constitutive equations for a piezoelectric material with crystal symmetry class $\mathrm{C}_{6 \mathrm{v}}$ can be expressed as follows. 


$$
\begin{gathered}
\left\{\begin{array}{l}
\varepsilon_{r} \\
\varepsilon_{\theta} \\
\varepsilon_{z} \\
\gamma_{\theta z} \\
\gamma_{z r} \\
\gamma_{r \theta}
\end{array}\right\}=\left[\begin{array}{cccccc}
s_{11}^{E} & s_{12}^{E} & s_{13}^{E} & 0 & 0 & 0 \\
s_{12}^{E} & s_{11}^{E} & s_{13}^{E} & 0 & 0 & 0 \\
s_{13}^{E} & s_{13}^{E} & s_{33}^{E} & 0 & 0 & 0 \\
0 & 0 & 0 & s_{44}^{E} & 0 & 0 \\
0 & 0 & 0 & 0 & s_{44}^{E} & 0 \\
0 & 0 & 0 & 0 & 0 & s_{66}^{E}
\end{array}\right]\left\{\begin{array}{l}
\sigma_{r} \\
\sigma_{\theta} \\
\sigma_{z} \\
\tau_{\theta z} \\
\tau_{z r} \\
\tau_{r \theta}
\end{array}\right\}+\left[\begin{array}{ccc}
0 & 0 & d_{31} \\
0 & 0 & d_{31} \\
0 & 0 & d_{33} \\
0 & d_{15} & 0 \\
d_{15} & 0 & 0 \\
0 & 0 & 0
\end{array}\right]\left\{\begin{array}{l}
E_{r} \\
E_{\theta} \\
E_{z}
\end{array}\right\} \\
\left\{\begin{array}{l}
D_{r} \\
D_{\theta} \\
D_{z}
\end{array}\right\}=\left[\begin{array}{cccccc}
0 & 0 & 0 & 0 & d_{15} & 0 \\
0 & 0 & 0 & d_{15} & 0 & 0 \\
d_{31} & d_{31} & d_{33} & 0 & 0 & 0
\end{array}\right]\left\{\begin{array}{l}
\sigma_{r} \\
\sigma_{\theta} \\
\sigma_{z} \\
\gamma_{\theta z} \\
\gamma_{z r} \\
\gamma_{r \theta}
\end{array}\right\}+\left[\begin{array}{ccc}
\varepsilon_{11}^{T} & 0 & 0 \\
0 & \varepsilon_{11}^{T} & 0 \\
0 & 0 & \varepsilon_{33}^{T}
\end{array}\right]\left\{\begin{array}{l}
E_{r} \\
E_{\theta} \\
E_{z}
\end{array}\right\}
\end{gathered}
$$

where $\sigma_{r}, \sigma_{\theta}, \sigma_{z}, \tau_{\theta z}, \tau_{z r}, \tau_{\theta r}$ are the components of the stress, $\varepsilon_{r}, \varepsilon_{\theta}, \varepsilon_{z}, \gamma_{\theta z}, \gamma_{z r}, \gamma_{\theta r}$ are the components of the strain, and all the components are functions of $r, \theta, z$, and $t . s_{11}{ }^{E}, s_{12}{ }^{E}, s_{13}{ }^{E}$, $s_{33}{ }^{E}, s_{44}{ }^{E}, s_{66}{ }^{E}$ are the compliance constants, $d_{15}, d_{31}, d_{33}$ are the piezoelectric constants, $\varepsilon_{11}{ }^{T}, \varepsilon_{33}{ }^{T}$ are the dielectric constants, $D_{r}, D_{\theta}, D_{z}$ are the components of the electrical displacement, and $E_{r}, E_{\theta}, E_{z}$ are the components of the electrical field. The piezoelectric material is isotropic in the plane normal to the $\mathrm{z}$-axis. The charge equation of electrostatics is represented as:

$$
\frac{\partial D_{r}}{\partial r}+\frac{1}{r} \frac{\partial D_{\theta}}{\partial \theta}+\frac{1}{r} D_{r}+\frac{\partial D_{z}}{\partial z}=0
$$

The electric field-electric potential relations are given by:

$$
E_{r}=-\frac{\partial \varphi}{\partial r}, E_{\theta}=-\frac{1}{r} \frac{\partial \varphi}{\partial \theta}, E_{z}=-\frac{\partial \varphi}{\partial z}
$$

where $\varphi$ is the electrical potential. The differential equations of equilibrium for threedimensional problems in cylindrical coordinates are:

$$
\frac{\partial \sigma_{r}}{\partial r}+\frac{1}{r} \frac{\partial \tau_{r \theta}}{\partial \theta}+\frac{\partial \tau_{z r}}{\partial z}+\frac{\sigma_{r}-\sigma_{\theta}}{r}=\rho \frac{\partial^{2} u_{r}}{\partial t^{2}}
$$




$$
\begin{gathered}
\frac{\partial \tau_{r \theta}}{\partial r}+\frac{1}{r} \frac{\partial \sigma_{\theta}}{\partial \theta}+\frac{\partial \tau_{\theta z}}{\partial z}+\frac{2 \tau_{r \theta}}{r}=\rho \frac{\partial^{2} u_{\theta}}{\partial t^{2}}, \\
\frac{\partial \tau_{z r}}{\partial r}+\frac{1}{r} \frac{\partial \tau_{\theta z}}{\partial \theta}+\frac{\partial \sigma_{z}}{\partial z}+\frac{\tau_{z r}}{r}=\rho \frac{\partial^{2} u_{z}}{\partial t^{2}},
\end{gathered}
$$

where $u_{r}(r, \theta, z, t), u_{\theta}(r, \theta, z, t), u_{z}(r, \theta, z, t)$ are the displacements of the ring in the radial, tangential, and transverse direction, respectively. And $\rho$ is the material density. The straindisplacement relations for three-dimensional problems in cylindrical coordinates are given by:

$$
\begin{gathered}
\varepsilon_{r}=\frac{\partial u_{r}}{\partial r}, \varepsilon_{\theta}=\frac{u_{r}}{r}+\frac{1}{r} \frac{\partial u_{\theta}}{\partial \theta}, \varepsilon_{z}=\frac{\partial u_{z}}{\partial z}, \\
\gamma_{r \theta}=\frac{1}{r} \frac{\partial u_{r}}{\partial \theta}+\frac{\partial u_{\theta}}{\partial r}-\frac{u_{\theta}}{r}, \\
\gamma_{\theta z}=\frac{\partial u_{\theta}}{\partial z}+\frac{1}{r} \frac{\partial u_{z}}{\partial \theta}, \\
\gamma_{z r}=\frac{\partial u_{z}}{\partial r}+\frac{\partial u_{r}}{\partial z} .
\end{gathered}
$$

Because the piezoelectric disk is thin and the deformation is small, the kirchoff assumption is made. The kirchoff assumptions are as follows:

$$
\begin{gathered}
u_{r}(r, \theta, z, t)=u_{0}(r, \theta, t)+z \frac{\partial w_{0}(r, \theta, t)}{\partial r}, \\
u_{\theta}(r, \theta, z, t)=v_{0}(r, \theta, t)+\frac{z}{r} \frac{\partial w_{0}(r, \theta, t)}{\partial r}, \\
u_{z}(r, \theta, z, t)=w_{0}(r, \theta, t),
\end{gathered}
$$

where $u_{0}, v_{0}, w_{0}$ represent the radial, the tangential, and the transverse displacements of the middle surface of the plane, respectively. After inserting (6)-(8) into (5a), (5b), the straindisplacement relations can be obtained as: 


$$
\begin{gathered}
\varepsilon_{r}=\frac{\partial u_{r}}{\partial r}=\frac{\partial u_{0}}{\partial r}+z \frac{\partial^{2} w_{0}}{\partial r^{2}} \\
\varepsilon_{\theta}=\frac{u_{r}}{r}+\frac{1}{r} \frac{\partial u_{\theta}}{\partial \theta}=\frac{u_{0}}{r}+\frac{1}{r} \frac{\partial v_{0}}{\partial \theta}+z\left[\frac{\partial w_{0}}{\partial r}+\frac{\partial}{\partial \theta}\left(\frac{1}{r} \frac{\partial w_{0}}{\partial \theta}\right)\right], \\
\gamma_{r \theta}=\frac{1}{r} \frac{\partial u_{r}}{\partial \theta}+\frac{\partial u_{\theta}}{\partial r}-\frac{u_{\theta}}{r}=\frac{1}{r} \frac{\partial u_{0}}{\partial \theta}+\frac{\partial v_{0}}{\partial r}-\frac{v_{0}}{r} \\
+\frac{z}{r} \frac{\partial^{2} w_{0}}{\partial r \partial \theta}+z \frac{\partial}{\partial r}\left(\frac{1}{r} \frac{\partial w_{0}}{\partial \theta}\right)-\frac{z}{r^{2}} \frac{\partial w_{0}}{\partial \theta}
\end{gathered}
$$

Since the ring is thin, stress $\sigma_{z}$ can be neglected relative to the other stresses, and strain $\gamma_{\theta z}, \gamma_{z r}$ can also be neglected. Thus, the constitutive equations of (1a), (1b) can be simplified as:

$$
\begin{gathered}
\sigma_{r}=\frac{\varepsilon_{r}+v \varepsilon_{\theta}}{s_{11}^{E}\left(1-v^{2}\right)}-\frac{d_{31}}{s_{11}^{E}(1-v)} E_{z}, \\
\sigma_{\theta}=\frac{v \varepsilon_{r}+\varepsilon_{\theta}}{s_{11}^{E}\left(1-v^{2}\right)}-\frac{d_{31}}{s_{11}^{E}(1-v)} E_{z}, \\
\tau_{r \theta}=\frac{\gamma_{r \theta}}{2 s_{11}^{E}(1+v)}, \\
D_{z}=d_{31}\left(\sigma_{r}+\sigma_{\theta}\right)+\varepsilon_{33}^{T} E_{z},
\end{gathered}
$$

where $v$ is the Poisson's ratio. In the piezoelectric transformer, the radial extensional vibration can be generated by driving the input electrode with AC voltage. The radial extensional vibration is supposed to be axisymmetric, and the radial extensional displacement of the middle plane can be assumed to be:

$$
u_{r}(r, t)=U(r) e^{i \omega t}
$$

The stress-displacement relations for the extensional vibration are given by:

$$
\sigma_{r}=\frac{1}{s_{11}^{E}\left(1-v^{2}\right)}\left[\frac{d U}{d r}+v \frac{U}{r}\right]-\frac{d_{31} E_{z}}{s_{11}^{E}(1-v)}
$$




$$
\sigma_{\theta}=\frac{1}{s_{11}^{E}\left(1-v^{2}\right)}\left[v \frac{d U}{d r}+\frac{U}{r}\right]-\frac{d_{31} E_{z}}{s_{11}^{E}(1-v)}
$$

Substituting (15),(16) into (4a), the governing equation of extensional vibrations can be obtained:

$$
\frac{d^{2} U}{d r^{2}}+\frac{1}{r} \frac{d U}{d r}-\frac{U}{r^{2}}-\rho \omega^{2} s_{11}^{E}\left(1-v^{2}\right) U=0
$$

The general solution of (17) is:

$$
U(r)=C_{1} J_{1}(\beta r)+C_{2} Y_{1}(\beta r)
$$

where $J_{1}$ is the Bessel function of first kind and first order, $Y_{1}$ is the Bessel function of second kind and first order, and

$$
\beta^{2}=\rho s_{11}^{E}\left(1-v^{2}\right) \omega^{2}
$$

Because the stress-free boundary conditions must be satisfied at $r=R_{i}$ and $r=R_{o}$.

$$
\int_{-h / 2}^{h / 2} \sigma_{r} d z=0
$$

Thus, the constants $A$ and $B$ can be found in (21) and (22).

$$
\begin{gathered}
A=\frac{E_{z} d_{31}(1+v) R_{o}}{\Delta_{1}}\left[\beta R_{i} Y_{0}\left(\beta R_{o}\right)-\alpha(1-v) Y_{1}\left(\beta R_{o}\right)-\beta R_{i} Y_{0}\left(\beta R_{i}\right)+(1-v) Y_{1}\left(\beta R_{i}\right)\right], \\
B=\frac{E_{z} d_{31}(1+v) R_{o}}{\Delta_{1}}\left[\beta R_{i} J_{0}\left(\beta R_{i}\right)-(1-v) J_{1}\left(\beta R_{i}\right)-\beta R_{i} J_{0}\left(\beta R_{o}\right)+\alpha(1-v) J_{1}\left(\beta R_{o}\right)\right],
\end{gathered}
$$

where $\alpha=R_{i} / R_{o}$ and $\Delta_{1}$ is as follows.

$$
\begin{gathered}
\Delta_{1}=\left[\beta R_{i} J_{0}\left(\beta R_{i}\right)-(1-v) J_{1}\left(\beta R_{i}\right)\right]\left[\beta R_{o} Y_{0}\left(\beta R_{o}\right)-(1-v) Y_{1}\left(\beta R_{o}\right)\right] \\
-\left[\beta R_{i} Y_{0}\left(\beta R_{i}\right)-(1-v) Y_{1}\left(\beta R_{i}\right)\right]\left[\beta R_{o} J_{0}\left(\beta R_{o}\right)-(1-v) J_{1}\left(\beta R_{o}\right)\right]
\end{gathered}
$$

\subsection{Impedance of the Piezoelectric Transformer}

In the output part of the PT, the output electrical current $I_{o}$ for extensional vibrations can be developed as: 


$$
\begin{aligned}
& I_{o}=\frac{\partial}{\partial t} \int_{S_{o}} D_{z} d s \\
& =j \omega \int_{0}^{2 \pi} \int_{R_{i}}^{R_{1}}\left\{\frac{d_{31}(1+v)}{s_{11}^{E}\left(1-v^{2}\right)}\left[\frac{d U}{d r}+\frac{U}{r}\right]+\varepsilon_{33}^{T}\left(1-k_{p}^{2}\right) E_{z}\right\} r d r d \theta \\
& \quad=j \omega \pi \varepsilon_{33}^{T} E_{z} \cdot \frac{(1+v) k_{p}^{2} \Delta_{2}-\left(k_{p}^{2}-1\right)\left(R_{1}^{2}-R_{o}^{2}\right) \Delta_{1}}{\Delta_{1}} \\
& \quad \begin{aligned}
\Delta_{2} & =\beta R_{i} R_{o}\left[Y_{0}\left(\beta R_{o}\right)-Y_{o}\left(\beta R_{i}\right)\right]\left[R_{1} J_{1}\left(\beta R_{1}\right)-R_{i} J_{1}\left(\beta R_{i}\right)\right] \\
& +\beta R_{i} R_{o}\left[J_{0}\left(\beta R_{i}\right)-J_{o}\left(\beta R_{o}\right)\right]\left[R_{1} Y_{1}\left(\beta R_{1}\right)-R_{i} Y_{1}\left(\beta R_{i}\right)\right] \\
- & (1-v)\left[R_{i} Y_{1}\left(\beta R_{o}\right)-R_{o} Y_{1}\left(\beta R_{i}\right)\right]\left[R_{1} J_{1}\left(\beta R_{1}\right)-R_{i} J_{1}\left(\beta R_{i}\right)\right] \\
- & (1-v)\left[R_{o} J_{1}\left(\beta R_{i}\right)-R_{i} J_{1}\left(\beta R_{o}\right)\right]\left[R_{1} Y_{1}\left(\beta R_{1}\right)-R_{i} Y_{1}\left(\beta R_{i}\right)\right]
\end{aligned}
\end{aligned}
$$

From (24), the resonant frequencies can be determined when the output current $I_{o}$ approaches infinity. The characteristic equation of resonant frequencies for extensional vibrations is given by:

$$
\Delta_{1}=0
$$

In the input part of the PT, the input electrical current $I_{i}$ for extensional vibrations can be developed as:

$$
\begin{aligned}
I_{i}= & \frac{\partial}{\partial t} \int_{S_{i}} D_{z} d s \\
= & j \omega \int_{0}^{2 \pi} \int_{R_{2}}^{R_{o}}\left\{\frac{d_{31}(1+v)}{S_{11}^{E}\left(1-v^{2}\right)}\left[\frac{d U}{d r}+\frac{U}{r}\right]+\varepsilon_{33}^{T}\left(1-k_{p}^{2}\right) E_{z}\right\} r d r d \theta \\
& =j \omega \pi \varepsilon_{33}^{T} E_{z} \cdot \frac{(1+v) k_{p}^{2} \Delta_{3}-\left(k_{p}^{2}-1\right)\left(R_{o}^{2}-R_{2}^{2}\right) \Delta_{1}}{\Delta_{1}} \\
& \quad+\beta R_{i} R_{o}\left[J_{0}\left(\beta R_{i}\right)-J_{o}\left(\beta R_{o}\right)\right]\left[R_{o} Y_{1}\left(\beta R_{o}\right)-R_{2} Y_{1}\left(\beta R_{2}\right)\right] \\
- & (1-v)\left[R_{i} Y_{1}\left(\beta R_{o}\right)-R_{o} Y_{1}\left(\beta R_{i}\right)\right]\left[R_{o} J_{1}\left(\beta R_{o}\right)-R_{2} J_{1}\left(\beta R_{2}\right)\right] \\
- & (1-v)\left[R_{o} J_{1}\left(\beta R_{i}\right)-R_{i} J_{1}\left(\beta R_{o}\right)\right]\left[R_{o} Y_{1}\left(\beta R_{o}\right)-R_{2} Y_{1}\left(\beta R_{2}\right)\right]
\end{aligned}
$$


From (27), the resonant frequencies can be determined when the input current $I_{i}$ approaches infinity. The characteristic equation of resonant frequencies can be obtained, which is the same with (26). It is noted that the resonant frequencies of the PT can be obtained based on the measured impedance spectrum, and the same results will be obtained in spite of the measured electrodes are in the input part or in the output part. According to (19) and (26), the resonant frequencies for ring-type PT can be expressed as:

$$
f=\frac{\beta}{2 \pi \sqrt{\rho s_{11}^{E}\left(1-v^{2}\right)}}
$$

\section{Electromechanical Model}

\subsection{Electromechanical Model of the PT}

The PT is not only a mechanical system but also electrical system. In this section, the electromechanical model for piezoelectrically coupled electromechanical systems will be derived. From Hagood's paper (Hagood, 1990), we have a generalized form of Hamilton's principle for a coupled electromechanical system:

$$
\int_{t_{1}}^{t_{2}} \partial\left[T-U+W_{1}+W_{2}\right] d t=0
$$

where $T$ is the kinetic energy, $U$ is the potential energy of the system, $W_{1}$ is the applied electric energy in the driving portion, and $W_{2}$ is the applied electric energy in the receiving portion. $T, U, W_{1}, W_{2}$ can be written as

$$
\begin{gathered}
T=\frac{1}{2} \int_{0}^{h} \int_{0}^{2 \pi} \int_{R_{i}}^{R_{o}} \rho \dot{u}_{r}^{2}(r, t) r d r d \theta d z \\
U=\frac{1}{2} \int_{0}^{h} \int_{0}^{2 \pi} \int_{R_{i}}^{R_{o}}\left[S^{T} T+E^{T} D\right] r d r d \theta d z \\
\partial W_{1}=-\partial \varphi_{i} \cdot q_{i}, \\
\partial W_{2}=-\partial \varphi_{o} \cdot q_{o},
\end{gathered}
$$

where $\rho$ is the density of the piezoelectric material. $\varphi_{i}$ and $q_{i}$ are the electric potential and the applied charge in the driving portion, respectively. $\varphi_{0}$ and $q_{i}$ are the electric potential and the applied charge in the receiving portion. By substituting Eqs.(31)-(34) into Eq.(30), the equations of motion for the PT can be written in Laplace transform as 


$$
\begin{gathered}
\left(m_{n} s^{2}+d_{n} s+k_{n}\right) X+A_{o} V_{o}=A_{i} V_{i}, \\
s A_{i} X+s C_{i} V_{i}=I_{i}, \\
s A_{o} X=s C_{o} V_{o}+I_{o},
\end{gathered}
$$

where $V_{i}$ and $I_{i}$ represent the input voltage and current in the driving portion, $V_{o}$ and $I_{o}$ represent the output voltage and current in the receiving port. The mass $m_{n}$, the stiffness $k_{n}$, input turn ratio $A_{i}$, output turn ratio $A_{o}$ for the equivalent circuit of piezoelectric transformer can be obtained from the follows.

$$
\begin{gathered}
m_{n}=\int_{0}^{h} \int_{0}^{2 \pi} \int_{R_{i}}^{R_{o}} \rho U^{2}(r) r d r d \theta d z \\
k_{n}=\int_{0}^{h} \int_{0}^{2 \pi} \int_{R_{i}}^{R_{o}}\left[c_{11}^{E}\left(\frac{\partial U}{\partial r}\right)^{2}+2 c_{12}^{E} \frac{\partial U}{\partial r} \frac{U}{r}+c_{22}^{E} \frac{U^{2}}{r^{2}}\right] r d r d \theta d z \\
C_{o}=\int_{0}^{h} \int_{0}^{2 \pi} \int_{R_{i}}^{R_{1}} \varepsilon_{33}^{T}\left(\frac{\partial \varphi}{\partial z}\right)^{2} r d r d \theta d z \\
C_{i}=\int_{0}^{h} \int_{0}^{2 \pi} \int_{R_{2}}^{R_{o}} \varepsilon_{33}^{T}\left(\frac{\partial \varphi}{\partial z}\right)^{2} r d r d \theta d z \\
A_{o}=\int_{0}^{h} \int_{0}^{2 \pi} \int_{R_{i}}^{R_{1}} e_{31} \frac{\partial \varphi}{\partial z} \cdot\left[\frac{\partial U}{\partial r}+\frac{U}{r}\right] r d r d \theta d z \\
A_{i}=\int_{0}^{h} \int_{0}^{2 \pi} \int_{R_{2}}^{R_{o}} e_{31} \frac{\partial \varphi}{\partial z} \cdot\left[\frac{\partial U}{\partial r}+\frac{U}{r}\right] r d r d \theta d z
\end{gathered}
$$

According to Eqs.(35)-(37), equivalent circuit model of the PT is shown in Fig.3. From the equivalent circuit model, we can see that Eq.(35) satisfy Kirchhoff's voltage law equation, which shows that the input voltage $A_{i} V_{i}$ is the sum of the output voltage $A_{o} V_{o}$ and the voltage difference $\left(m_{n} s^{2}+d_{n} s+k_{n}\right)$ X. Eq.(36) satisfy Kirchhoff's current law equation in the driving portion, which shows that the input current $I_{i}$ is the sum of the current flowing through $\left(m_{n} s^{2}+d_{\mathrm{n}} \mathrm{s}+k_{n}\right)$ and the current flowing through $C_{i}$. Eq.(37) satisfy Kirchhoff's current law equation in the receiving portion, which shows that the current flowing through $\left(m_{n} s^{2}+d_{n} s+k_{n}\right)$ is the sum of the current flowing through $C_{o}$ and the output current $I_{o}$. 


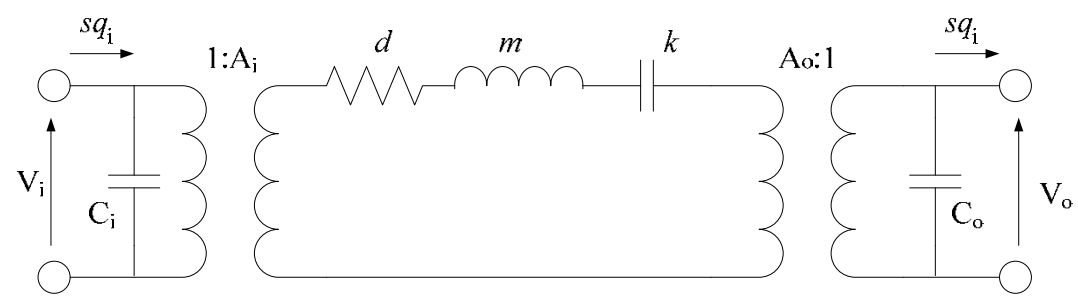

Fig. 3. Equivalent circuit of the piezoelectric transformer.

\subsection{Characteristics of the PT}

There is no output current in the receiving portion when the electrodes are open-circuited. Thus, voltage step-up ratio for the PT can be obtained based on Eqs.(35)(37). Substituting $I_{o}$ $=0$ into Eq.(37) and eliminating $X(s)$ from Eqs. (35)(37) gives

$$
\frac{V_{o}(s)}{V_{i}(s)}=\frac{A_{o} A_{i}}{\left(m_{n} s^{2}+d_{n} s+k_{n}\right) C_{o}+A_{o}^{2}} .
$$

When a load resistance $R_{L}$ is connected between the electrodes in the receiving portion of the PT, Eq.(45) can be obtained by substituting $I_{o}=V_{o} / R_{L}$ into Eq.(37).

$$
s A_{o} X=s C_{o} V_{o}+V_{o} / R_{L}
$$

The voltage step-up ratio for the PT with a load resistance $R_{L}$ in the receiving portion can be obtained based on Eqs.(35)(45) as the following.

$$
\frac{V_{o}(s)}{V_{i}(s)}=\frac{s A_{i} A_{o} R_{L}}{\left(m_{n} s^{2}+d_{n} s+k_{n}\right)\left(s C_{o} R_{L}+1\right)+s A_{o}^{2} R_{L}}
$$

If the electrodes in the receiving portion of the PT is short-circuited, the voltage step-up ratio for the PT can be obtained as zero by substituting $R_{L}=0$ into Eq.(46). In addition, Eq.(46) shows that the higher the load resistance $R_{L}$, the higher the voltage step-up ratio. The maximum voltage step-up ratio can be obtained as Eq.(44) when the load resistance $R_{L}$ approach infinite. On the other hand, the output power of the PT can be calculated by the power consumption of the load resistance $R_{L}$ as the following:

$$
P_{o}=\left|V_{o}\right|^{2} / R_{L}
$$

If the natural frequency is chosen as the operating frequency in the PT, then the voltage step-up ratio can be rewritten as 


$$
\frac{V_{o}}{V_{i}}=\frac{A_{i} A_{o}}{j \omega_{n} C_{o} d_{n}+d_{n} / R_{L}+A_{o}}
$$

Therefore, the output power of the PT can be obtained by substituting Eq.(48) into Eq.(47).

$$
P_{o}=\left|V_{o}\right|^{2} / R_{L}=\frac{A_{i}^{2} A_{o}^{2} V_{i}^{2}}{R_{L}\left[\left(\omega_{n} C_{o} d_{n}\right)^{2}+\left(d_{n} / R_{L}+A_{o}\right)^{2}\right]}
$$

According to equivalent circuit of the PT shown as in Fig.3, the input power of the PT can be calculated by the sum of the power consumption of the damping $d_{n}$ and that of the load resistance $R_{L}$. Eq.(37) shows that the current flowing through $d_{n}$ is $\left(s C_{o} V_{o}+I_{o}\right) / A_{o}$, thus the input power of the PT can be obtained as

$$
\begin{gathered}
P_{i}=\frac{d_{n}}{A_{o}^{2}}\left|j \omega_{n} C_{o} V_{o}+V_{o} / R_{L}\right|^{2}+P_{o} \\
=V_{o}^{2}\left[d_{n}\left(\omega_{n} C_{o} / A_{o}\right)^{2}+d_{n} /\left(R_{L} A_{o}^{2}\right)+1 / R_{L}\right] .
\end{gathered}
$$

Therefore, the efficiency of the PT can be obtained as

$$
\eta=\frac{P_{o}}{P_{i}}=\frac{1}{d_{n} R_{L}\left(\omega_{n} C_{o} / A_{o}\right)^{2}+d_{n} /\left(A_{o}^{2} R_{L}\right)+1}
$$

The maximum efficiency can be calculated by the differential of Eq.(50). Thus, the maximum efficiency can be obtained when the optimal load resistance $R_{L, o p t}$ is

$$
R_{L, o p t}=1 /\left(\omega_{n} C_{o}\right)
$$

Substituting Eq.(52) into Eq.(51) gives the maximum efficiency.

$$
\eta_{\max }=\frac{A_{o}^{2}}{2 d_{n} \omega_{n} C_{o}+A_{o}^{2}}
$$

It is note that the smaller the damping coefficient $d_{n}$, the higher the maximum efficiency.

\section{Simulation and Experiment}

\subsection{Experimental Setup and the Impedance Measurements}

To verify the electromechanical model, a ring-type PT with $16 \mathrm{~mm}$ in outer diameter, $8 \mathrm{~mm}$ in inner diameter, and $1 \mathrm{~mm}$ in thickness was used. The PT is has silver electrodes on two 
opposite surfaces and is poled along its thickness direction. One of the electrodes of the PT is split into two regions on the diameter of $11 \mathrm{~mm}$. The transformer structure was fabricated using the piezoelectric material APC 840 by APC International, USA. The material properties provided by the supplier are listed in Table I. The displacement distributions of the mode shapes based on theoretical analysis for the PT are presented in Fig.4. Also, to easily realize the dynamic behavior of the PT, a finite element method analysis of the vibration of the PT is conducted. And the results of the extensional vibration modes of the PT are shown in Fig.5(a)(b)(c).

A HP 4194A Impedance Analyzer was used to measure the input impedance and output impedance, and results are shown in Fig.6. The input impedance was measured for the shorted electrodes in the receiving portion, and the output impedance was measured for the shorted electrodes in the driving portion. This transformer was designed to operate in the first vibration mode. For the input impedance of the PT, the first resonant frequency is 91.2 $\mathrm{kHz}$, the first anti-resonant frequency is $94.05 \mathrm{kHz}$. For the output impedance of the PT, the first resonant frequency is $91.2 \mathrm{kHz}$, the first anti-resonant frequency is $93.6 \mathrm{kHz}$ in the input impedance of the PT. It shows that nearly the same resonant frequency were obtained in spite of the impedance was measured from the driving portion or the receiving portion. The results are the same with theoretical analysis of Eqs. (24) and (27).

Basd on Eqs.(34)-(36), input impedance as a function of frequency at different load resistances are calculated and shown in Fig.7. And the experimental results are shown in Fig.8. In the input impedance of the PT with load resistance varied from short $\left(R_{L}=0\right)$ to open $\left(R_{L}=\infty\right)$, it shows that the peak frequency is changed from $94.05 \mathrm{kHz}$ to $97.85 \mathrm{kHz}$. The peak frequency is increased as the load resistance is increased. Also, there exists an optimal load resistance $R_{L, o p t}$, which shows the maximum damping ratio in the input impedance when compared with the other different load resistances. We can also calculated the optimal load resistance $R_{L, o p t}=2.6 \mathrm{k} \Omega$ from Eq.(52). It should be noted that efficiency of the PT approaches to the maximum efficiency when the load resistance $R_{L}$ approaches the optimal load resistance $R_{L, o p t}$.

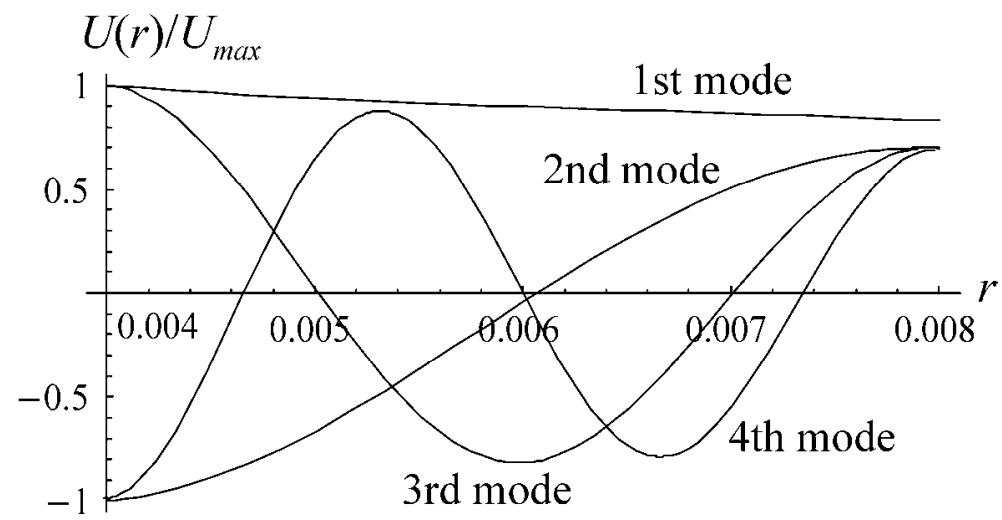

Fig. 4. Mode shapes of the piezoelectric transformer. 


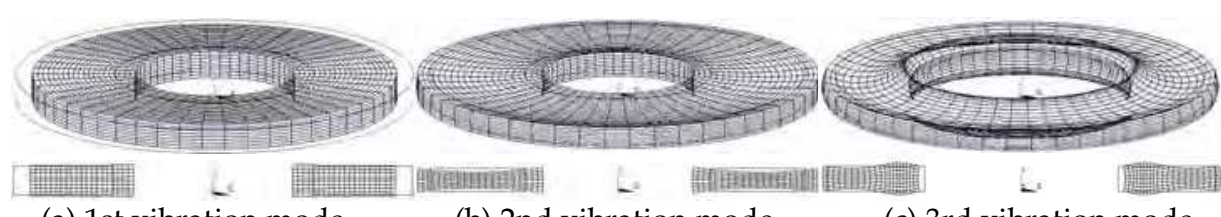
(a) 1st vibration mode
(b) 2nd vibration mode
(c) 3rd vibration mode

Fig. 5. Vibration modes of piezoelectric transformer.

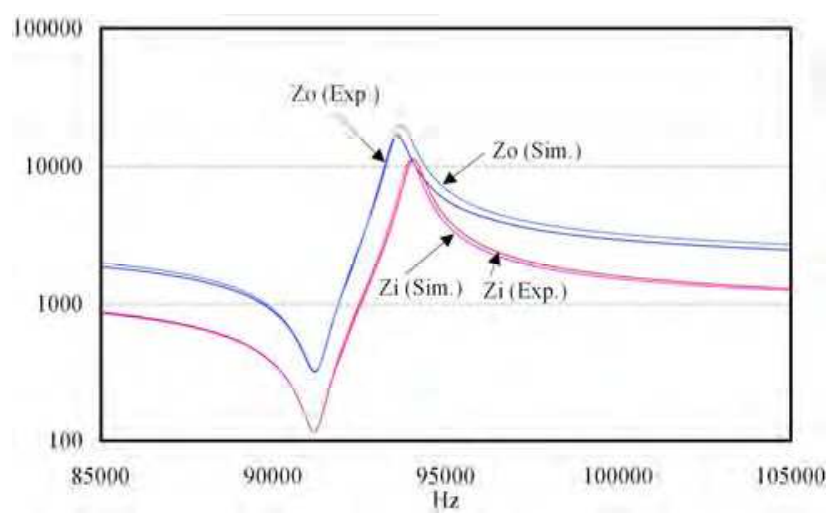

Fig. 6. Input and output impedance

\subsection{Voltage Step-up Ratio, Output Power, and Efficiency}

The experimental setup for the measurement of the voltage step-up ratio and output power of the PT is illustrated in Fig.9. A function generator (NF Corporation, WF1943) and a high frequency amplifier (NF Corporation, HSA4011) were used for driving power supply. The variation in electric characteristics with load resistance and driving frequency were measured with a multi-meter (Agilent 34401A). The voltage step-up ratios as a function of frequency at different load resistances were measured and compared with theoretical analysis, as shown in Fig.10. It shows that the experimental results are in a good agreement with the theoretical results, so the proposed electromechanical model for the PT was verified.

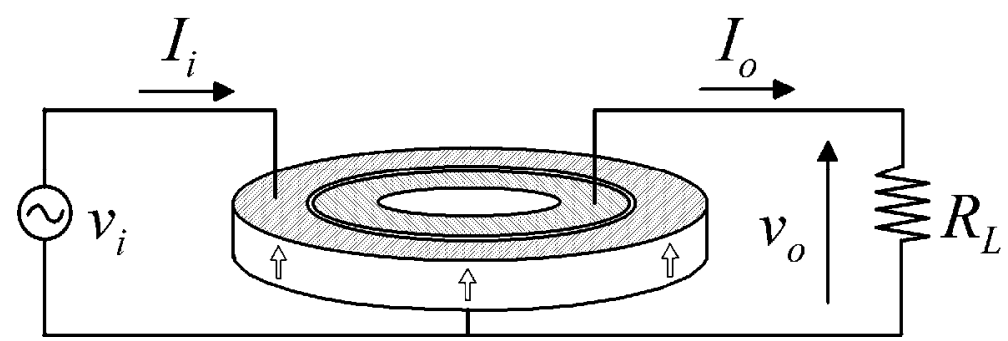

Fig. 7. Experimental setup 


\begin{tabular}{|l|l|}
\hline Piezoelectric coefficient $d_{31}$ & $-125 \times 10^{-12} \mathrm{C} / \mathrm{N}$ \\
\hline Coupling factor $k_{\mathrm{p}}$ & 0.59 \\
\hline Mechanical quality factor $Q_{\mathrm{m}}$ & 500 \\
\hline Dielectric constant $\varepsilon_{33} / \varepsilon_{0}$ & 1694 \\
\hline Density $\rho$ & $7600 \mathrm{~g} / \mathrm{cm}^{3}$ \\
\hline Young's modulus $\mathrm{Y}_{11^{\mathrm{E}}}$ & $8 \times 10^{10} \mathrm{~N} / \mathrm{m}^{2}$ \\
\hline
\end{tabular}

Table 1. Properties of piezoelectric material.

\begin{tabular}{|l|l|}
\hline Input piezoelectric capacitance $C_{i}$ & $1.5 \mathrm{nF}$ \\
\hline Output piezoelectric capacitance $C_{o}$ & $671.5 \mathrm{pF}$ \\
\hline Input turn ratio $A_{i}$ & 0.1198 \\
\hline Output turn ratio $A_{o}$ & 0.07545 \\
\hline Effective mass $m_{1}$ & $4.773 \times 10^{-4} \mathrm{~kg}$ \\
\hline Effective damping $d_{1}$ & $1.868 \mathrm{~N}-\mathrm{s} / \mathrm{m}$ \\
\hline Effective stiffness $k_{1}$ & $1.569 \times 10^{8} \mathrm{~N} / \mathrm{m}$ \\
\hline
\end{tabular}

Table 2. Parameters of the equivalent circuit
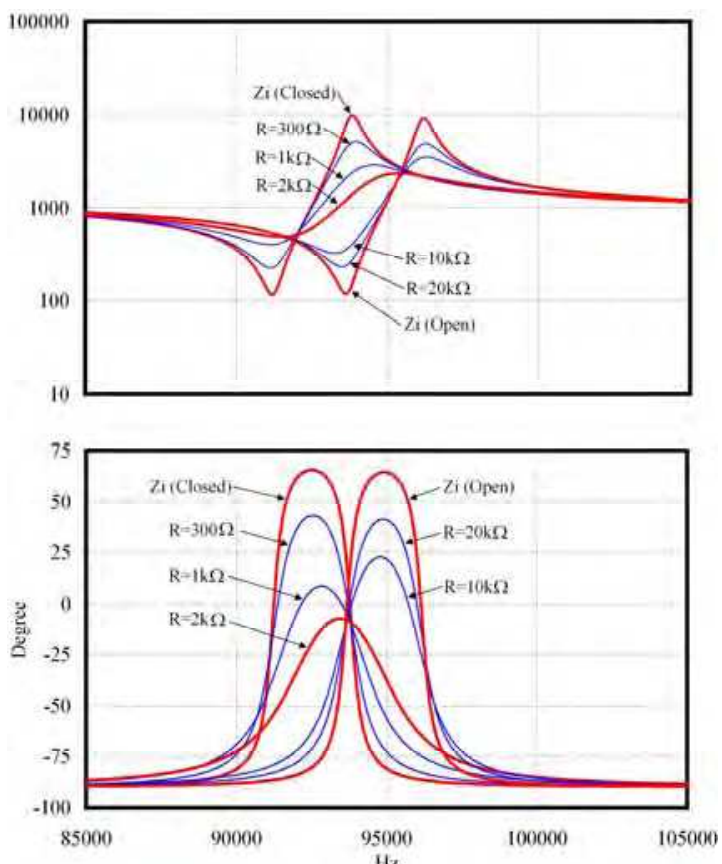

Fig. 8. Calculated input impedance 

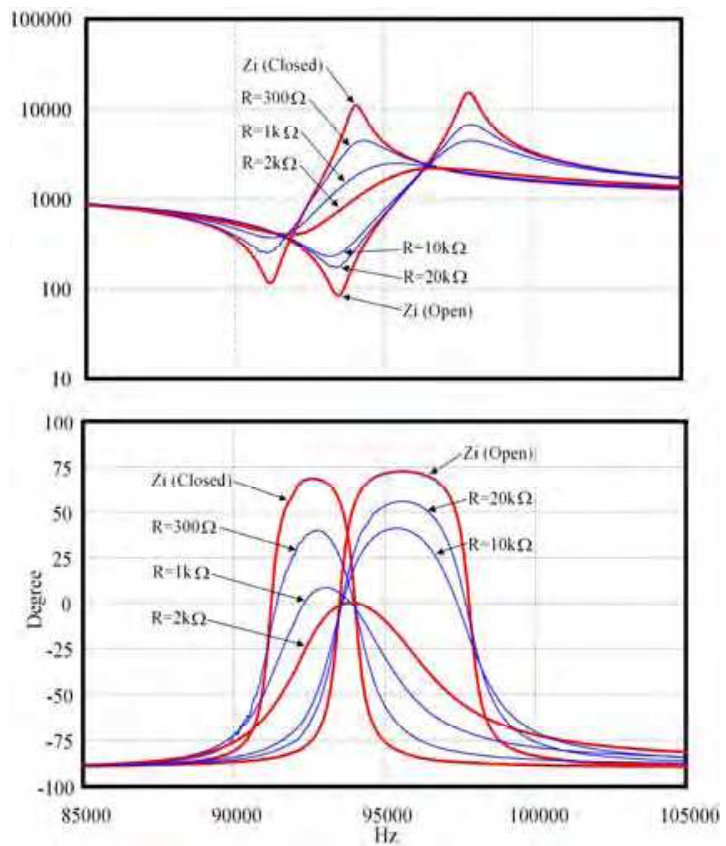

Fig. 9. Measured input impedance

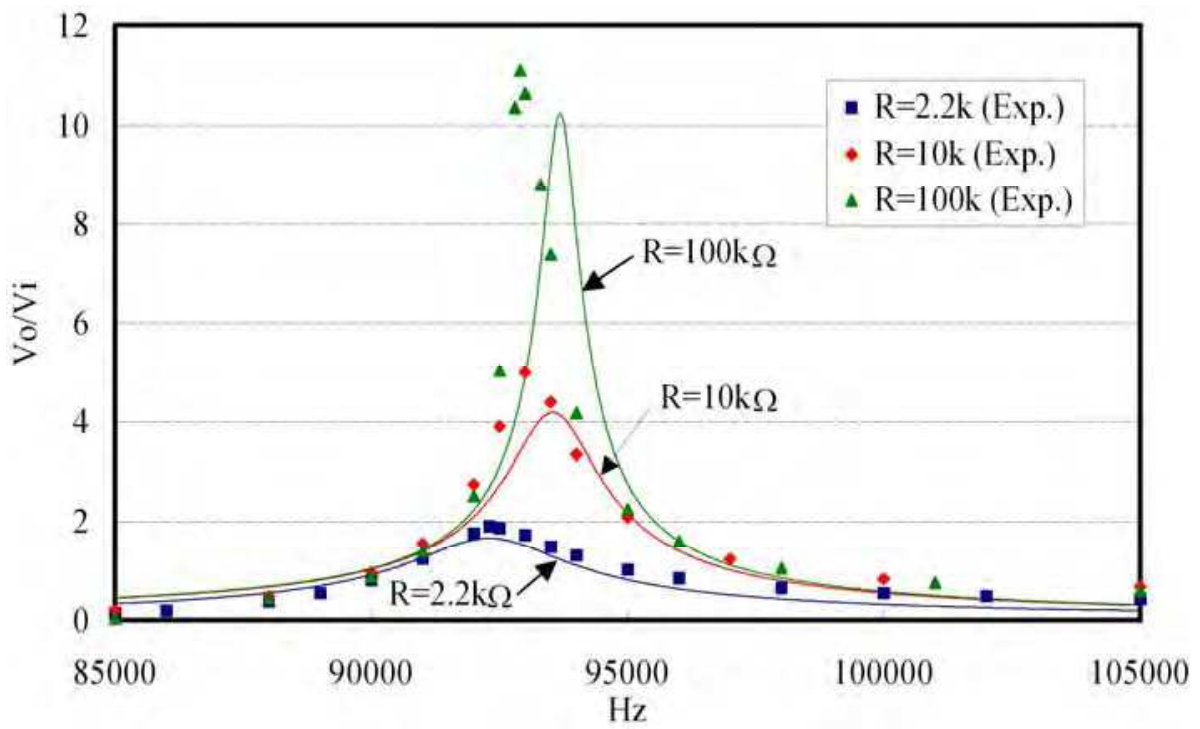

Fig. 10. Voltage step-up ratio 


\section{Conclusion}

In this chapter, an electromechanical model for ring-type PT is presented. An equivalent circuit of the PT is shown based on the electromechanical model. Also, the voltage step-up ratio, input impedance, output impedance, and output power of the PT are calculated, and the optimal load resistance and the maximum efficiency for the PT have been obtained. In the last, some simulated results of the electromechanical model are compared with the experimental results for verification. The model presented here lays foundation for a general framework capable of serving a useful design tool for optimizing the configuration of the PT.

\section{References}

Bishop, R. P. (1998). Multi-Layer Piezoelectric Transformer, US Patent No.5834882.

Hagood, N. W. Chung, W. H. Flotow, A. V. (1990). Modeling of Piezoelectric Acatuator Dynamics for Active Structural Control. Intell. Mater. Syst. And Struct., Vol.1, pp. 327-354, ISSN:1530-8138.

Hu, J. H. Li, H. L. Chan, H. L. W. Choy, C. L. (2001). A Ring-shaped Piezoelectric Transformer Operating in the third Sysmmetric Extenxional Vibration Mode. Sensors and Actuators, A., No.88, pp. 79-86, ISSN:0924-4247.

Laoratanakul, P. Carazo, A. V. Bouchilloux P. Uchino, K. (2002). Unipoled Disk-type Piezoelectric Transformers. Jpn. J. Appl. Phys., Vol.41, No., pp. 1446-1450, ISSN:1347-4065.

Rosen, C. A. (1956). Ceramic Transformers and Filters, Proceedings of Electronic Comp., pp. 205-211.

Sasaki, Y. Uehara, K. Inoue, T. (1993). Piezoelectric Ceramic Transformer Being Driven with Thickness Extensional Vibration, US Patent No.5241236. 


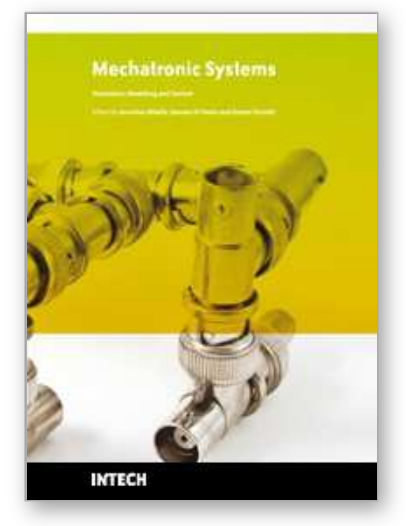

\author{
Mechatronic Systems Simulation Modeling and Control \\ Edited by Annalisa Milella Donato Di Paola and Grazia Cicirelli
}

ISBN 978-953-307-041-4

Hard cover, 298 pages

Publisher InTech

Published online 01, March, 2010

Published in print edition March, 2010

This book collects fifteen relevant papers in the field of mechatronic systems. Mechatronics, the synergistic blend of mechanics, electronics, and computer science, integrates the best design practices with the most advanced technologies to realize high-quality products, guaranteeing at the same time a substantial reduction in development time and cost. Topics covered in this book include simulation, modelling and control of electromechanical machines, machine components, and mechatronic vehicles. New software tools, integrated development environments, and systematic design methods are also introduced. The editors are extremely grateful to all the authors for their valuable contributions. The book begins with eight chapters related to modelling and control of electromechanical machines and machine components. Chapter 9 presents a nonlinear model for the control of a three-DOF helicopter. A helicopter model and a control method of the model are also presented and validated experimentally in Chapter 10. Chapter 11 introduces a planar laboratory testbed for the simulation of autonomous proximity manoeuvres of a uniquely control actuator configured spacecraft. Integrated methods of simulation and Real-Time control aiming at improving the efficiency of an iterative design process of control systems are presented in Chapter 12. Reliability analysis methods for an embedded Open Source Software (OSS) are discussed in Chapter 13. A new specification technique for the conceptual design of self-optimizing mechatronic systems is presented in Chapter 14 . Chapter 15 provides a general overview of design specificities including mechanical and control considerations for micro-mechatronic structures. It also presents an example of a new optimal synthesis method to design topology and associated robust control methodologies for monolithic compliant microstructures.

\title{
How to reference
}

In order to correctly reference this scholarly work, feel free to copy and paste the following:

Shine-Tzong Ho (2010). Electromechanical Analysis of a Ring-type Piezoelectric Transformer, Mechatronic Systems Simulation Modeling and Control, Annalisa Milella Donato Di Paola and Grazia Cicirelli (Ed.), ISBN: 978-953-307-041-4, InTech, Available from: http://www.intechopen.com/books/mechatronic-systemssimulation-modeling-and-control/electromechanical-analysis-of-a-ring-type-piezoelectric-transformer

\section{INTECH}

open science | open minds

\author{
InTech Europe \\ University Campus STeP Ri \\ Slavka Krautzeka 83/A
}

\section{InTech China}

Unit 405, Office Block, Hotel Equatorial Shanghai

No.65, Yan An Road (West), Shanghai, 200040, China 
51000 Rijeka, Croatia

Phone: +385 (51) 770447

Fax: +385 (51) 686166

www.intechopen.com
中国上海市延安西路65号上海国际贵都大饭店办公楼 405 单元 Phone: +86-21-62489820

Fax: +86-21-62489821 
(C) 2010 The Author(s). Licensee IntechOpen. This chapter is distributed under the terms of the Creative Commons Attribution-NonCommercialShareAlike-3.0 License, which permits use, distribution and reproduction for non-commercial purposes, provided the original is properly cited and derivative works building on this content are distributed under the same license. 